\title{
Inhalation of hydrogen gas attenuates ouabain- induced auditory neuropathy in gerbils
}

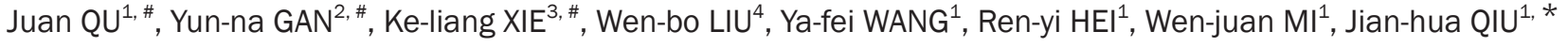 \\ ${ }^{1}$ Department of Otolaryngology, Xijing Hospital, Fourth Military Medical University, Xi'an 710032, China; ${ }^{2}$ Department of Prosthondon- \\ tics, College of Stomatology, Fourth Military Medical University, Xi'an 710032, China; ${ }^{3}$ Department of Anesthesiology, General Hospital \\ of Tianjin Medical University, Tianjin 300052, China; ${ }^{4}$ Department of Neurosurgery, Xijing Institute of Clinical Neuroscience, Xijing Hos- \\ pital, Fourth Military Medical University, Xi'an 710032, China
}

\begin{abstract}
Aim: Auditory neuropathy (AN) is a hearing disorder characterized by abnormal auditory nerve function with preservation of normal cochlear hair cells. This study was designed to investigate whether treatment with molecular hydrogen $\left(\mathrm{H}_{2}\right)$, which can remedy damage in various organs via reducing oxidative stress, inflammation and apoptosis, is beneficial to ouabain-induced AN in gerbils.

Methods: AN model was made by local application of ouabain $(1 \mathrm{mmol} / \mathrm{L}, 20 \mathrm{~mL})$ to the round window membrane in male Mongolian gerbils. $\mathrm{H}_{2}$ treatment was given twice by exposing the animals to $\mathrm{H}_{2}(1 \%, 2 \%$, and $4 \%$ ) for $60 \mathrm{~min}$ at $1 \mathrm{~h}$ and $6 \mathrm{~h}$ after ouabain application. Before and $7 \mathrm{~d}$ after ouabain application, the hearing status of the animals was evaluated using the auditory brainstem response (ABR) approach, the hear cell function was evaluated with distortion product otoacoustic emissions (DPOAE). Seven days after ouabain application, the changes in the cochleae, especially the spiral ganglion neurons (SGNs), were morphologically studied. TUNEL staining and immunofluorescent staining for activated caspase-3 were used to assess the apoptosis of SGNs.

Results: Treatment with $\mathrm{H}_{2}$ (2\% and 4\%) markedly attenuated the click and tone burst-evoked ABR threshold shift at 4, 8 , and $16 \mathrm{kHz}$ in ouabain-exposed animals. Neither local ouabain application, nor $\mathrm{H}_{2}$ treatment changed the amplitude of DPOAE at 4, 8, and $16 \mathrm{kHz}$. Morphological study showed that treatment with $\mathrm{H}_{2}(2 \%)$ significantly alleviated SGN damage and attenuated the loss of SGN density for each turn of cochlea in ouabain-exposed animals. Furthermore, ouabain caused significantly higher numbers of apoptotic SGNs in the cochlea, which was significantly attenuated by the $\mathrm{H}_{2}$ treatment. However, ouabain did not change the morphology of cochlear hair cells.

Conclusion: The results demonstrate that $\mathrm{H}_{2}$ treatment is beneficial to ouabain-induced AN via reducing apoptosis. Thus, $\mathrm{H}_{2}$ might be a potential agent for treating hearing impairment in AN patients.
\end{abstract}

Keywords: auditory neuropathy (AN); ouabain; hearing loss; hydrogen gas $\left(\mathrm{H}_{2}\right)$; cochlea; spiral ganglion neuron; hair cell; auditory brainstem response; distortion product otoacoustic emissions; apoptosis

Acta Pharmacologica Sinica (2012) 33: 445-451; doi: 10.1038/aps.2011.190; published online 5 Mar 2012

\section{Introduction}

Auditory neuropathy (AN), also known as auditory dys-synchrony $(\mathrm{AD})$, is a sensorineural hearing disorder and accounts for $7 \%-10 \%$ of all permanent childhood hearing impairment ${ }^{[1]}$. It was recently identified to define a specific type of hearing deficit resulting from the impairment of auditory nerve function $^{[2]}$. Two hallmark features of AN are normal outer hair cell function and the presence of absent/abnormal auditory brainstem responses $(\mathrm{ABR})^{[3]}$. Unfortunately, there is no effective treatment for AN mainly because its mechanism is unclear ${ }^{[3]}$.

\footnotetext{
\# These three authors contributed equally to this work.

* To whom correspondence should be addressed.

E-mail qiujh@fmmu.edu.cn

Received 2011-08-19 Accepted 2011-12-09
}

In 2007, Ohsawa et al found that hydrogen gas $\left(\mathrm{H}_{2}\right)$ demonstrated an antioxidant effect by selectively reducing hydroxyl radicals $[\cdot \mathrm{OH}$, the most cytotoxic reactive oxygen species (ROS)] without interfering with other physiological $\operatorname{ROS}^{[4]}$. Recently, many studies, including ours, have found that $\mathrm{H}_{2}$ has therapeutic roles in many diseases via its ability to reduce oxidative stress, inflammation and apoptosis; these diseases include cerebral damage, spinal cord injury, sepsis, multiple organ dysfunction, chronic allograft nephropathy, tumors and type 2 diabetes ${ }^{[4-14]}$. Furthermore, $\mathrm{H}_{2}$ can effectively protect against the morphological and functional cochlear hair cell damage induced by $\mathrm{ROS}^{[15]}$. In addition, a more recent study has reported that pretreatment with $\mathrm{H}_{2}$ in drinking water can facilitate the recovery of hair cell function and attenuate noiseinduced temporary hearing loss ${ }^{[16]}$. These findings strongly 
indicate that $\mathrm{H}_{2}$ treatment may be beneficial in cases of AN.

Ouabain is a cardiac glycoside that can specifically bind to $\mathrm{Na}^{+} / \mathrm{K}^{+}$-ATPase and block its activity. Following application of ouabain into the round window (RW) niche of gerbil, the animals showed relatively normal otoacoustic emissions and cochlear microphonics in conjunction with increased thresholds for cochlear whole-nerve action potentials (CAPs) and $\mathrm{ABRs}^{[17-19]}$. The ouabain-induced AN model has been shown to selectively and permanently destroy most spiral ganglion neurons (SGNs) with little effect on the morphology and function of the sensory hair cells and the cells in the cochlear lateral wall ${ }^{[17-19]}$. Thus, the ouabain-exposed gerbil is believed to be a reliable model of human $\mathrm{AN}^{[17]}$. In the present study, we tested the hypothesis that $\mathrm{H}_{2}$ could attenuate ouabain-induced $\mathrm{AN}$ in gerbils by reducing apoptosis.

\section{Materials and methods Animals}

Male Mongolian gerbils aged 4-8 months with healthy external ears were used in this study. The animals, which had normal Preyer's reflexes, were provided by the animal center of the Capital Medical University in China. The animals were housed at $20-22^{\circ} \mathrm{C}$ with a 12-h light/dark cycle. Standard animal chow and water were freely available. All experimental procedures were approved by the Institutional Animal Care and Use Committee of Fourth Military Medical University and performed in accordance with the National Institutes of Health (NIH, USA) guidelines for the use of experimental animals.

The animals were randomly divided into four groups: Saline, Saline $+\mathrm{H}_{2}$, Ouabain and Ouabain $+\mathrm{H}_{2}$. The animals in the Ouabain and Ouabain $+\mathrm{H}_{2}$ groups were subjected to the ouabain-induced AN model. The $\mathrm{H}_{2}$ treatment was administered at $1 \mathrm{~h}$ and $6 \mathrm{~h}$ after ouabain or saline administration.

\section{Ouabain-induced AN model}

The ouabain-induced AN model was executed as described previously ${ }^{[17]}$. Briefly, the animals were anesthetized with sodium pentobarbital $(40 \mathrm{mg} / \mathrm{kg})$. Their body temperatures were measured and maintained at $37.5 \pm 0.5^{\circ} \mathrm{C}$ using a heating pad. Sterile procedures were used to apply ouabain to the RW, wherein $20 \mu \mathrm{L}$ of a $1 \mathrm{mmol} / \mathrm{L}$ ouabain (Sigma-Aldrich Chemical Co, St Louis, MO, USA) solution in normal saline was placed in the RW niche for $1 \mathrm{~h}$. Then, the ouabain was removed with a small piece of filter paper. Finally, the surface of the bulla was fully closed with dental cement, and the incisions were closed with sutures. The control animals received normal saline injections in equal volumes.

\section{$\mathrm{H}_{2}$ treatment}

Based on our previous studies ${ }^{[9,12,13]}, \mathrm{H}_{2}$ treatment was given via $1 \%, 2 \%$, or $4 \% \mathrm{H}_{2}$ inhalation for $60 \mathrm{~min}$ at $1 \mathrm{~h}$ and again at $6 \mathrm{~h}$ after ouabain or saline injection. The animals assigned to the $\mathrm{H}_{2}$ treatment were put in a sealed Plexiglas chamber with inflow and outflow outlets. $\mathrm{H}_{2}$ was supplied through a TF-I gas flowmeter (YUTAKA Engineering Corp, Tokyo, Japan) and delivered by air into the chamber at a rate of $4 \mathrm{~L} / \mathrm{min}$.
The concentration of $\mathrm{H}_{2}$ in the chamber was continuously monitored with a commercially available detector (Hy-Alerta Handheld Detector Model 500, $\mathrm{H}_{2}$ Scan, Valencia, CA, USA). The concentration of oxygen in the chamber was maintained at $21 \%$ with supplemental oxygen and continuously monitored with a gas analyzer (Medical Gas Analyzer LB-2, Model $40 \mathrm{~mol} / \mathrm{L}$, Beckman, USA). Carbon dioxide was removed from the chamber with Baralyme. The animals that were not assigned to receive the $\mathrm{H}_{2}$ treatment were exposed to room air in this chamber.

\section{Auditory brainstem response (ABR)}

The hearing status of all animals was evaluated at baseline just before and $7 \mathrm{~d}$ after ouabain or saline administration using the ABR approach as previously described ${ }^{[16]}$. Elevations of the ABR threshold have been shown to provide an excellent, reliable indicator of the degree of cochlear hearing loss for experimental animals ${ }^{[20]}$. The animals were anesthetized with an intraperitoneal injection of $40 \mathrm{mg} / \mathrm{kg}$ pentobarbital sodium, and needle electrodes were placed subcutaneously beneath the pinna of the measured ear (reference electrode), beneath the apex of the nose (ground) and at the vertex (active electrode). The stimulus signal was generated with an Intelligent Hearing Systems device (Bio-Logic Systems, USA). Click sounds were produced at a rate of $57.7 / \mathrm{s}$ to evoke the ABRs. Tone burst sounds at 4,8 , and $16 \mathrm{kHz}(0.2-\mathrm{ms}$ rise/fall time and 1-ms flat segment) were generated to estimate the frequency-specific thresholds. The responses of 1024 sweeps were averaged at each intensity level step. The intensity of the stimulus was varied at stepwise increments of $5 \mathrm{~dB}$ sound pressure levels (SPL), and the threshold was defined as the lowest intensity level at which a response was still observed. Rectal temperatures of the animals were monitored and maintained by a warming pad throughout the recordings.

\section{Distortion product otoacoustic emissions (DPOAE)}

As described previously ${ }^{[16]}$, the animals underwent DPOAE measurement at baseline just before and $7 \mathrm{~d}$ after ouabain or saline administration with an acoustic probe using the DP2000 DPOAE measurement system, version 3.0 (Starkey Laboratory, Eden Prairie, MN, USA). The DP-grams comprised 2f1-f2 DPOAE amplitudes as a function of $\mathrm{f} 2$. The stimulus parameters used for the DPOAE input/output (I/O) growth function were constructed as follows: a frequency ratio of $1.2\left(\mathrm{f}_{2} /\right.$ $f_{1}$ ) and $\mathrm{f} 2$ in one-sixth-octave steps from 1 to $16 \mathrm{kHz}$. DPOAE was defined as being present when its level exceeded that of the noise floor by $3 \mathrm{~dB}$.

\section{Histological assessment}

The animals were euthanized by decapitation under deep anesthesia after the ABR test at $7 \mathrm{~d}$. The cochleae were quickly removed and fixed with $4 \%$ paraformaldehyde in $0.1 \mathrm{~mol} / \mathrm{L}$ phosphate-buffered saline (PBS, $\mathrm{pH} 7.4$ ) for $24 \mathrm{~h}$ at room temperature. After fixation, the cochleae were dissected and decalcified in 2\% ethylenediaminetetraacetic acid (EDTA) and $0.25 \%$ glutaraldehyde for 3 weeks. Then, they were sectioned 
at $6 \mu \mathrm{m}$ thickness and stained with hematoxylin and eosin. The sections were digitized using an Olympus BX51 microscope (Olympus Optical, Hamburg, Germany). To determine the spiral ganglion neuronal density for each turn in the cochlea, the area of each spiral ganglion was measured with a MetaMorph imaging system (Universal Imaging Corporation, Downingtown, PA, USA), and morphologically intact SGNs (criterion: round cell body containing a nucleus and homogenous cytoplasm) were counted within this area ${ }^{[17]}$.

\section{TUNEL staining}

To detect DNA fragmentation in the nuclei of cells, we used a TUNEL kit (Roche Diagnostics GmbH, Mannheim, Germany) to quantify the occurrence of apoptosis $24 \mathrm{~h}$ after ouabain or saline administration ${ }^{[9]}$. To determine the number of SGNs that underwent apoptosis, two independent and blinded pathologists counted the SGNs that were positive or negative in the TUNEL staining.

\section{Immunofluorescent staining for activated caspase-3}

The cochleae were harvested, fixed, and decalcified in $2 \%$ ETDA for 3 weeks. Then, the samples were sectioned at 6 $\mu \mathrm{m}$ thickness and stained with cleaved caspase-3 (Asp175) antibody (Cell Signaling Technology, Danvers, MA, USA) as previously described ${ }^{[17]}$. Photomicrographs were taken with a fluorescent microscope (Nikon Instruments, Melville, NY, USA). Microscopic examination was performed by at least two investigators, who were blinded to the sample identification.

\section{Caspase-3 activity}

Caspase-3 activity in the cochlear tissue was measured with a caspase-3/CPP32 Fluorometric Assay Kit (Biovision Research Products, Mountain View, CA, USA) $24 \mathrm{~h}$ after ouabain or saline administration ${ }^{[9]}$. All assays were run in duplicate.

\section{Statistical analysis}

All data are expressed as the mean \pm SEM. The data were analyzed by one-way ANOVA followed by an LSD- $t$ test for multiple comparisons. The statistical analysis was performed with SPSS (Statistical Package for the Social Sciences) 16.0 software. In all tests, a $P$ value less than 0.05 was considered statistically significant.

\section{Results}

\section{Click and tone burst-evoked ABR threshold shift}

In this study, we first investigated the effects of $\mathrm{H}_{2}$ treatment on hearing impairment in gerbils with ouabain-induced AN. The $\mathrm{H}_{2}$ treatment was given twice with $1 \%, 2 \%$, or $4 \%$ $\mathrm{H}_{2}$ inhalation for $60 \mathrm{~min}$ starting at $1 \mathrm{~h}$ and again at $6 \mathrm{~h}$ after ouabain or saline administration. Based on our preliminary experiment, hearing status was evaluated before and $7 \mathrm{~d}$ after ouabain or saline administration by measuring the click and tone burst-evoked ABR threshold shift; these data are shown in Figures 1 and 2. The administration of ouabain significantly increased the click-evoked ABR threshold and tone burst-

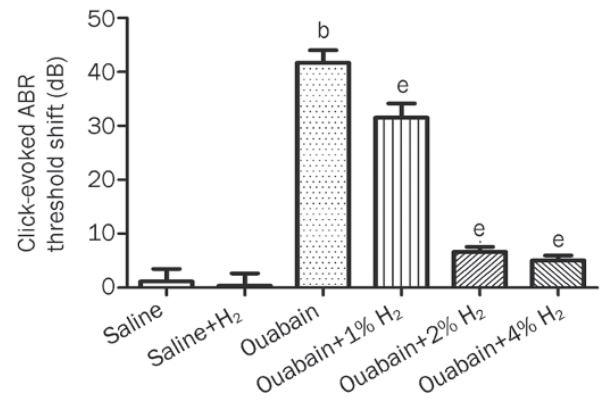

Figure 1. $\mathrm{H}_{2}$ treatment attenuated ouabain-induced click-evoked auditory brainstem response (ABR) threshold shift. $\mathrm{H}_{2}$ treatment was given twice with $1 \%, 2 \%$, or $4 \% \mathrm{H}_{2}$ inhalation for 60 min starting at $1 \mathrm{~h}$ and $6 \mathrm{~h}$ after ouabain or saline injection, respectively. The click-evoked ABR threshold was measured at baseline just before and again $7 \mathrm{~d}$ after ouabain or saline administration. The values are expressed as mean \pm SEM ( $n=6$ per group). ${ }^{b} P<0.05$ vs Saline group. ${ }^{e} P<0.05$ vs Ouabain group.

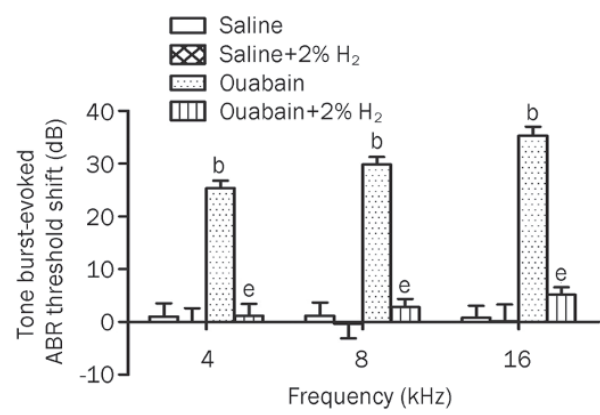

Figure 2. $\mathrm{H}_{2}$ treatment attenuated ouabain-induced tone burst-evoked auditory brainstem response (ABR) threshold shift. $\mathrm{H}_{2}$ treatment was given twice with $2 \% \mathrm{H}_{2}$ inhalation for 60 min starting at $1 \mathrm{~h}$ and $6 \mathrm{~h}$ after ouabain or saline injection, respectively. The tone burst-evoked ABR threshold was measured at baseline just before and again $7 \mathrm{~d}$ after ouabain or saline administration. The values are expressed as mean \pm SEM ( $n=6$ per group). ${ }^{b} P<0.05$ vs Saline group. ${ }^{e} P<0.05$ vs Ouabain group.

evoked ABR threshold at 4, 8, and $16 \mathrm{kHz}$ when compared with the saline group ( $P<0.05, n=6$ per group), suggesting that ouabain caused significant hearing impairment. However, the $\mathrm{H}_{2}$ treatment markedly attenuated the click and tone burstevoked ABR threshold shift at 4, 8, and $16 \mathrm{kHz}$ in ouabainexposed animals $\left(P<0.05, n=6\right.$ per group). In addition, the $\mathrm{H}_{2}$ treatment had no effects on the click and tone burst-evoked ABR threshold at 4,8 , and $16 \mathrm{kHz}$ in the animals with saline administration ( $P>0.05, n=6$ per group). These results demonstrate that local administration of ouabain causes a hearing disorder in gerbils, which can be significantly attenuated by $\mathrm{H}_{2}$ treatment.

\section{DPOAE audiograms}

We further investigated the effects of the $\mathrm{H}_{2}$ treatment on hair cell function in gerbils with ouabain-induced AN. Hair cell function was evaluated before and $7 \mathrm{~d}$ after ouabain or saline 
administration by distortion product otoacoustic emissions (DPOAE), which are shown in Figure 3. Here, we found that local administration of ouabain did not change the amplitude of DPOAE at 4,8 , and $16 \mathrm{kHz}(P>0.05$ vs Saline group, $n=6$ per group), which was not influenced by the $\mathrm{H}_{2}$ treatment $(P>0.05$ vs Ouabain group, $n=6$ per group). This result demonstrates that ouabain does not damage hair cell function, and $\mathrm{H}_{2}$ treatment also has no effects on hair cell function in this model.

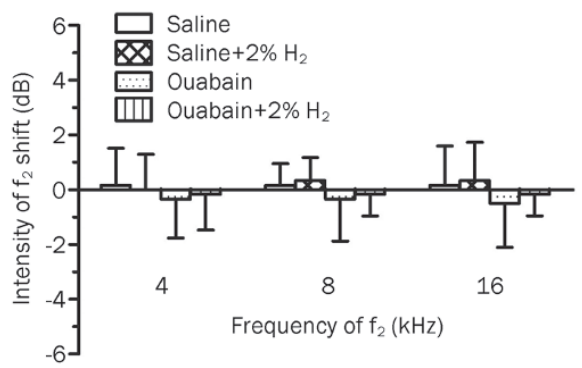

Figure 3. Distortion product otoacoustic emissions (DPOAE) measurements at $\mathrm{f}_{2}=4,8$, and $16 \mathrm{kHz}$. $\mathrm{H}_{2}$ treatment was given as Figure 2. The hear cell function was evaluated before and $7 \mathrm{~d}$ after ouabain or saline injection by DPOAE. The values are expressed as mean \pm SEM ( $n=6$ per group).

\section{Histological assessment}

Furthermore, we investigated the morphological changes in the cochleae, especially the SGNs, $7 \mathrm{~d}$ after ouabain or saline administration (Figure 4A-4D). Ouabain caused significant damage to the SGNs, such as edema, as well as the occurrence of vacuole-like structures, condensed pyknotic nuclei and shrinking of the cytoplasm. The $\mathrm{H}_{2}$-treated, ouabainexposed animals exhibited normal morphological profiles of the SGNs. In addition, we measured the spiral ganglion neuronal density for each turn in the cochlea (Figure 4E). Ouabain drastically decreased the SGN density in the basal, middle and apical turns in cochlea when compared with the saline group ( $P<0.05, n=6$ per group). However, the $\mathrm{H}_{2}$ treatment significantly increased the SGN density of the basal, middle and apical turns in the cochleae of the ouabain-exposed animals $(P<0.05, n=6$ per group). These results demonstrate that ouabain causes significant damage to the SGNs, which can be attenuated by $\mathrm{H}_{2}$ treatment.

In addition, we observed the morphology of the cochlear hair cells by staining with Alexa Fluor 488 conjugated to phalloidin (green fluorescence). There was no significant damage in the inner and outer hair cells of the cochleae in ouabainexposed animals (Figure 5).

\section{TUNEL staining and caspase- 3 activity}

In addition, we detected apoptosis of the SGNs $24 \mathrm{~h}$ after ouabain or saline administration by TUNEL staining and caspase-3 activity. The results are shown in Figures 6 and 7. TUNEL staining identified no apoptotic cells in the cochleae
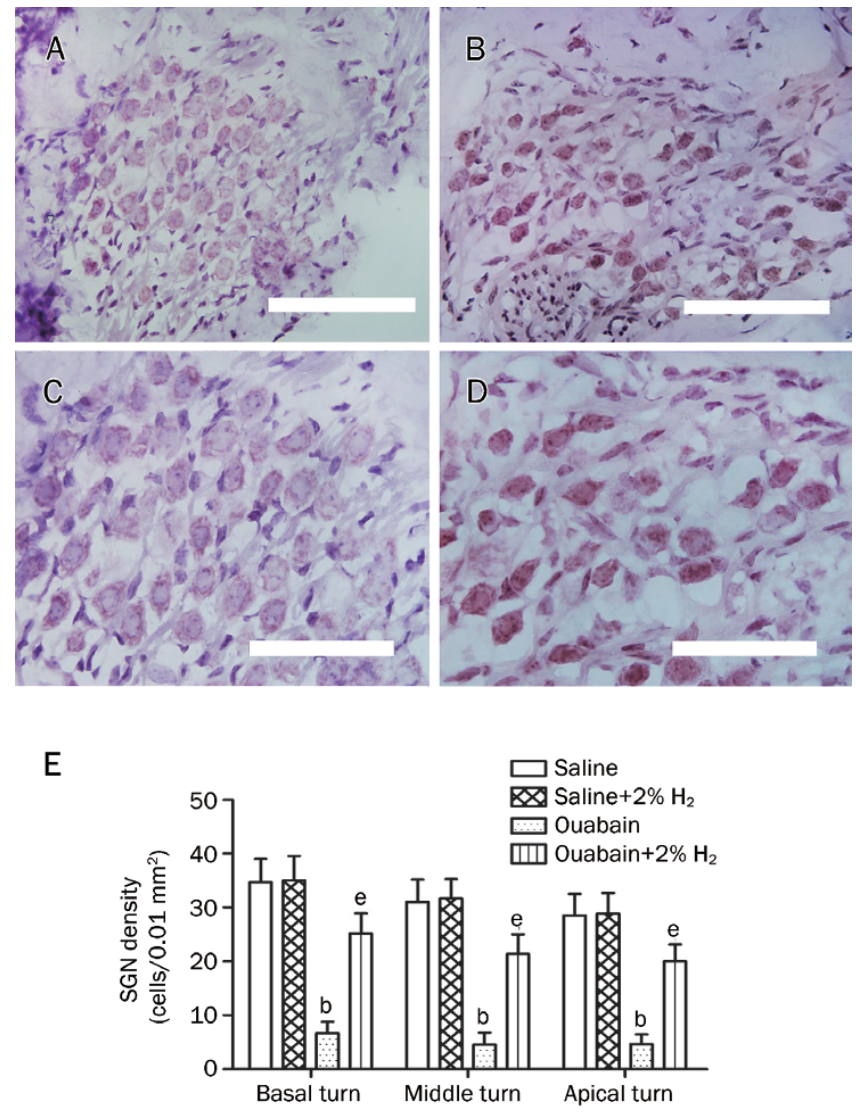

Figure 4. $\mathrm{H}_{2}$ treatment attenuated ouabain-induced damage of spiral ganglion neurons (SGN). $\mathrm{H}_{2}$ treatment was given as Figure 2. The SGN damage was evaluated with hematoxylin and eosin staining $7 \mathrm{~d}$ after ouabain or saline administration. Representative micrographs in the middle turn are shown in 4A-4D (A and C: Saline group; B and D: Ouabain group). $E$ is the quantitative analysis of spiral ganglion neuronal density for each turn in the cochlea. The values are expressed as mean \pm SEM ( $n=6$ per group). ${ }^{b} P<0.05$ vs Saline group. ${ }^{e} P<0.05$ vs Ouabain group. Scale bar for $A$ and $B$ is $50 \mu \mathrm{m}$; Scale bar for $C$ and $D$ is $30 \mu \mathrm{m}$.

of the saline-exposed animals. There was a high number of TUNEL-positive SGNs in the ouabain-treated animals, but these numbers were significantly lower in the animals receiving the $\mathrm{H}_{2}$ treatment. The number of SGNs that were positive for TUNEL staining was recorded in each specimen in a blind fashion. We found that ouabain-exposed animals had significantly more apoptotic SGNs $(P<0.05$ vs Saline group, $n=6$ per group). However, $\mathrm{H}_{2}$ treatment resulted in significantly fewer apoptotic SGNs in the ouabain-exposed animals $(P<0.05$ vs Ouabain group, $n=6$ per group). Furthermore, we found that the caspase- 3 activity in the cochlea was significantly greater in the ouabain-exposed animals than in the saline-only animals $(P<0.05$ vs Saline group, $n=6$ per group, Figure 7$)$. The $\mathrm{H}_{2}$-treated animals that had been exposed to ouabain had significantly less caspase- 3 activity than the animals exposed to ouabain alone ( $P<0.05$ vs Ouabain group, $n=6$ per group, Figure 7). This result was confirmed by immunofluorescent staining for cleaved caspase-3 (red) (Figure 7). The results suggest 

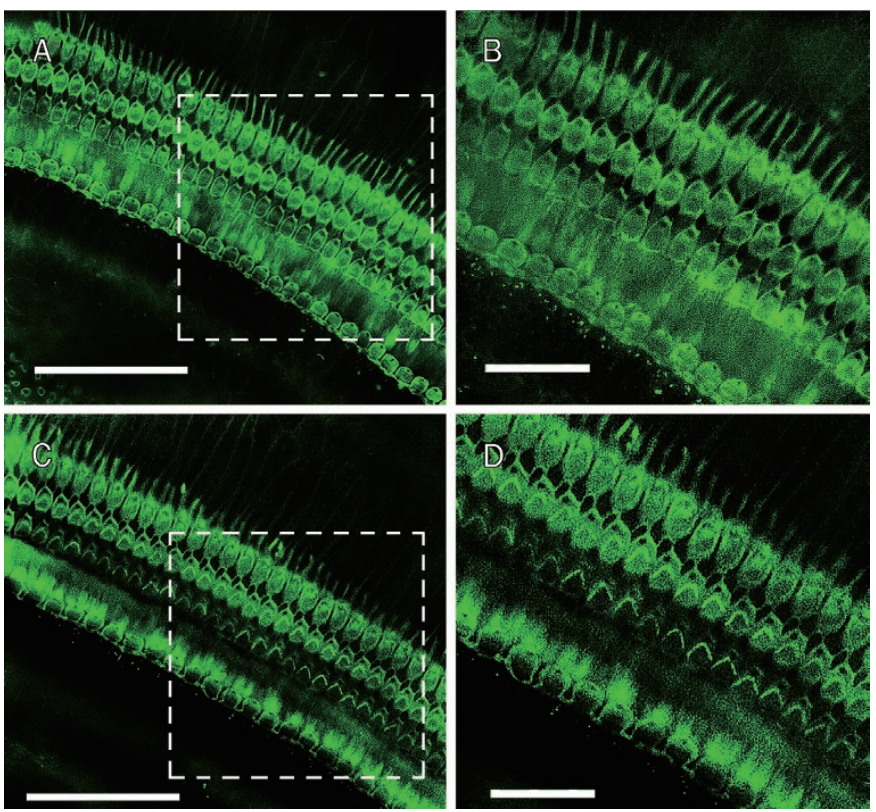

Figure 5. Morphology of cochlear hair cells in ouabain-exposed animals. The organ of corti was stained for filamentous actin with Alexa Fluor 488 conjugated to phalloidin (green fluorescence) (Molecular Probes, Eugene, OR, USA) for 40 min to outline hair cells ( $A$ and B: Saline group; C and D: Ouabain group). This figure showed that local administration of ouabain did not damage the outer and inner hair cells. Scale bar for $A$ and $C$ is 50 $\mu \mathrm{m}$; Scale bar for $B$ and $D$ is $15 \mu \mathrm{m}$.

that local administration of ouabain significantly increases the occurrence of cochlear SGN apoptosis, which can be alleviated by $\mathrm{H}_{2}$ treatment.

\section{Discussion}

In this study, we found that $\mathrm{H}_{2}$ treatment could reduce the click and tone burst-evoked ABR threshold shift induced by local administration of ouabain in gerbils. Furthermore, $\mathrm{H}_{2}$ treatment significantly attenuated ouabain-induced SGN damage and apoptosis. In addition, the local application of ouabain did not affect the morphology and function of the cochlear hair cells. These results strongly suggest that $\mathrm{H}_{2}$ might be a useful therapeutic agent for ouabain-induced AN through its capacity to inhibit apoptosis.

$\mathrm{AN}$ is a hearing disorder characterized by absent or severely impaired ABR in the presence of normal outer hair cell function and is evaluated by otoacoustic emissions and/or cochlear microphonics $^{[3]}$. Some possible sites of lesions that can produce the audiometric and electrophysiological profile of AN include: the inner hair cells, the synaptic junction between the inner hair cells and type I afferent nerve fibers, the SGNs and the specific damage or demyelinization of type I auditory nerve fibers ${ }^{[3]}$. The clinical features of AN can vary considerably with respect to the age of onset, etiology, severity of hearing loss and site of lesions ${ }^{[3]}$. Although the underlying mechanisms of AN are key to understanding and treating the disease, these mechanisms are still largely unknown.

In this study, we found that ouabain-exposed animals had significantly higher click and tone burst-evoked ABR thresholds. However, the local administration of ouabain did not change the amplitude of the DPOAE. In addition, the histological analysis showed that the local administration of ouabain led to significant damage to the SGNs and decreased the spiral ganglion neuronal density for each turn in the cochlea. However, ouabain did not damage the morphology of the cochlear hair cells. These results demonstrate that the local
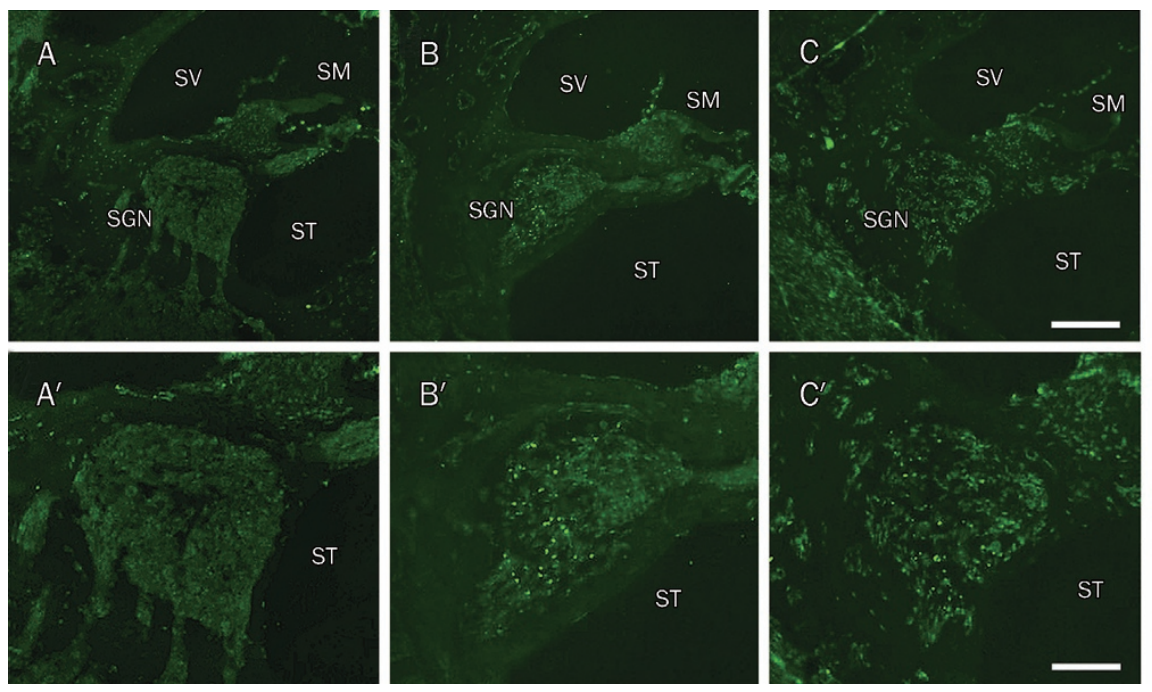

\section{D}

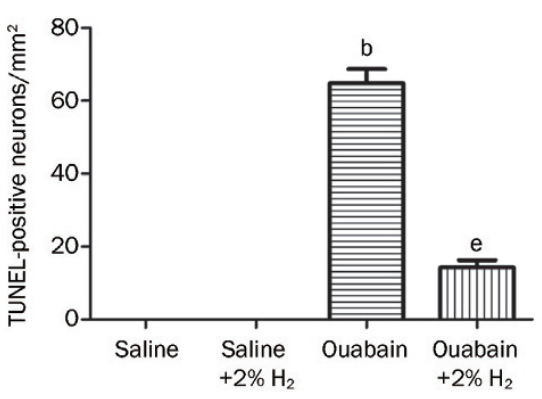

Figure 6. $\mathrm{H}_{2}$ treatment reduced the ouabain-induced cochlear SGN apoptosis evidenced by TUNEL staining. $\mathrm{H}_{2}$ treatment was given as Figure 2. The SGN apoptosis was detected by TUNEL staining $\left(A-C, A^{\prime}-C^{\prime}\right) 24 \mathrm{~h}$ after ouabain or saline administration. For quantitative measurement, the number of SGN that was positive for TUNEL staining (green) was recorded in each specimen in a blind fashion (D). The values are expressed as mean \pm SEM ( $n=6$ per group). ${ }^{b} P<0.05$ vs Saline group. ${ }^{e} P<0.05$ vs Ouabain group. ND=not detected. SGN, spiral ganglion neuron; SM, scala media; $S T$, scala tympani; SV, scala vestibuli. Scale bar for A, B, and C is $100 \mu \mathrm{m}$; Scale bar for $A^{\prime}, B^{\prime}$, and $C^{\prime}$ is $50 \mu \mathrm{m}$. 

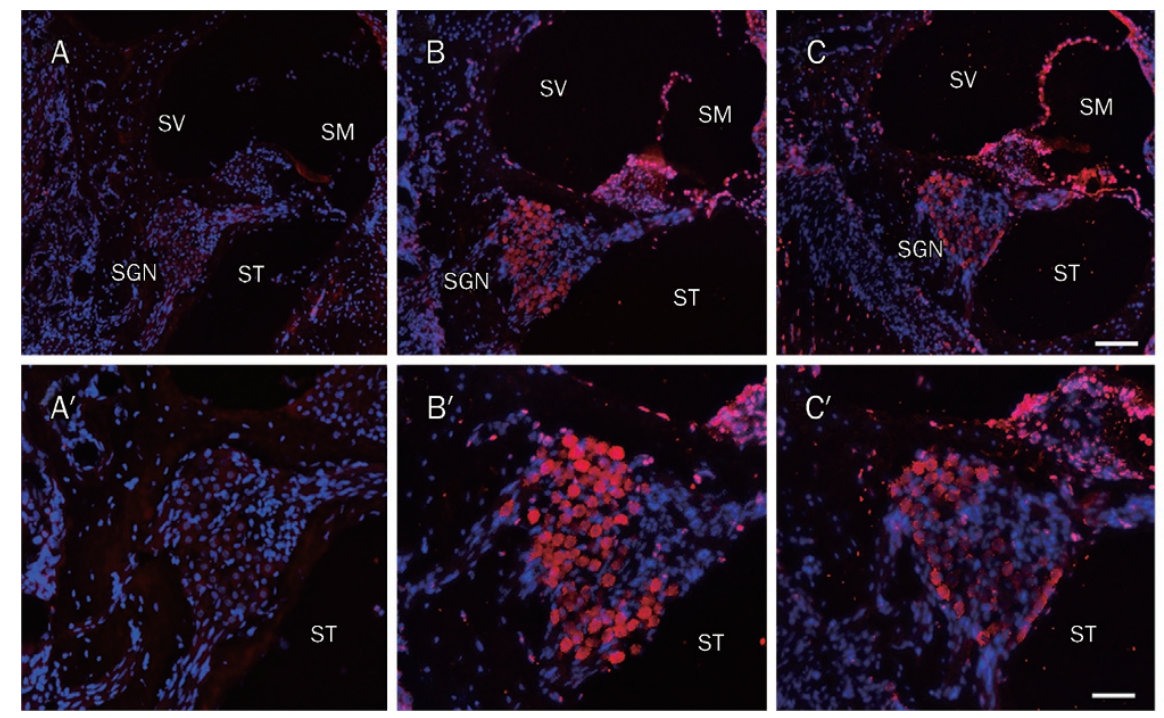

D

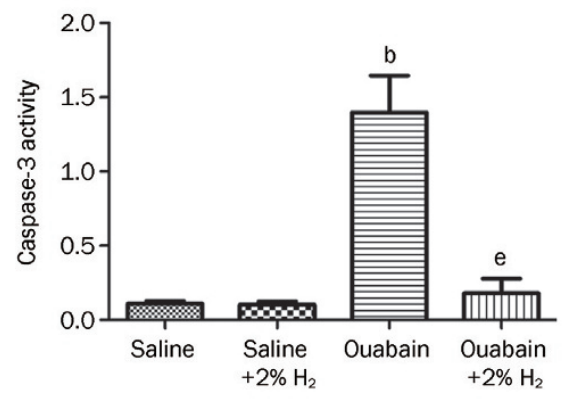

Figure 7. $\mathrm{H}_{2}$ treatment reduced the ouabain-induced cochlear SGN apoptosis evidenced by immunofluorescent staining for activated caspase-3 and measurement of caspase-3 activity. $\mathrm{H}_{2}$ treatment was given as Figure 2. The SGN apoptosis was detected by immunofluorescent staining for cleaved caspase-3 (A-C, $\left.A^{\prime}-C^{\prime}\right)$ as well as caspase-3 activity (D) $24 \mathrm{~h}$ after ouabain or saline administration. Positive signals are indicated by red fluorescence in contrast to blue fluorescence of Hoechst 33342-stained nuclei $\left(A-C, A^{\prime}-C^{\prime}\right)$. The caspase-3 activity is expressed as mean $\pm S E M$ ( $n=6$ per group). ${ }^{\mathrm{b}} P<0.05$ vs Saline group. ${ }^{\mathrm{e}} P<0.05$ vs Ouabain group. SGN, spiral ganglion neuron; SM, scala media; ST, scala tympani; SV, scala vestibule. Scale bar for A, $B$, and $C$ is $100 \mu \mathrm{m}$; Scale bar for $A^{\prime}, B^{\prime}$, and $C^{\prime}$ is $50 \mu \mathrm{m}$.

administration of ouabain successfully induced the AN model in gerbils in this study, which is consistent with previous studies $^{[17-19]}$.

Recently, we and other researchers have found that $\mathrm{H}_{2}$ treatment could effectively protect against damage to organs such as the brain, spinal cord, heart, lungs, liver and kidneys through reducing oxidative stress, inflammation and apoptosis. These findings suggest that $\mathrm{H}_{2}$ could play a role in preventive and therapeutic applications for organ damage ${ }^{[4-13,21,22]}$. Furthermore, an in vitro study has demonstrated that $\mathrm{H}_{2}$ can protect both the inner and outer hair cells from oxidant damage induced by different concentrations of antimycin $\mathrm{A}^{[15]}$. Incubation with a hydrogen-saturated medium also significantly reduced ROS generation and subsequent lipid peroxidation in the auditory epithelia, leading to the increased survival of hair cells ${ }^{[15]}$. In addition, a more recent study has shown that pretreatment with $\mathrm{H}_{2}$-rich water can prevent noise-induced hearing loss ${ }^{[16]}$. In the present study, we found that $\mathrm{H}_{2}$ treatment significantly attenuated the ouabain-induced increase in the click and tone burst-evoked ABR threshold. In addition, $\mathrm{H}_{2}$ treatment significantly mitigated damage to the SGNs and prevented a decreased spiral ganglion neuronal density for each turn of the cochlea in ouabain-exposed animals. Also, $\mathrm{H}_{2}$ treatment did not change the morphology and function of cochlear hair cells. These results demonstrate that $\mathrm{H}_{2}$ treatment is beneficial for ouabain-induced AN.

The degeneration of SGNs is a common pathologic feature correlated with permanent hearing loss. SGN apoptosis plays an important role in the ouabain-induced AN model ${ }^{[18]}$. Apoptosis is a programmed cell death that is characterized by specific ultrastructural changes including cell shrink- age, nuclear condensation and DNA fragmentation ${ }^{[23]}$. It has been reported that the application of ouabain to the RW membrane of the cochlea results in a rapid loss of SGNs, as indicated both by TUNEL staining and direct observation of the nuclear morphology ${ }^{[17]}$. In addition, ouabain-induced AN in gerbils is associated with the release of cytochrome $c$ and the activation of caspase-3 in SGN ${ }^{[18]}$. In the present study, we also found that the local administration of ouabain resulted in significant levels of cochlear SGN apoptosis as evidenced by TUNEL staining and caspase- 3 activity, which could be significantly attenuated by $\mathrm{H}_{2}$ treatment. Several previous studies have reported that $\mathrm{H}_{2}$ has an anti-apoptotic role in many diseases ${ }^{[4,5,9]}$. Therefore, the results suggest that the protective roles of $\mathrm{H}_{2}$ treatment may be associated with reduced SGN apoptosis. In addition, $\mathrm{H}_{2}$ treatment can reduce the levels of oxidative products and inflammatory cytokines, as well as increase antioxidant enzymatic activities in many diseases ${ }^{[4,6,8-14]}$. Further studies are needed to determine whether these mechanisms contribute to the protective effects of $\mathrm{H}_{2}$ treatment against AN.

$\mathrm{H}_{2}$ is one of the most plentiful gasses in the universe. It is neither explosive nor dangerous at a concentration of less than $4.7 \%$ in air and $4.1 \%$ in pure oxygen. This study supports the observation that $\mathrm{H}_{2}$ inhalation may be an effective therapeutic agent for attenuating ouabain-induced $\mathrm{AN}$ via the reduction of SGN apoptosis. Therefore, $\mathrm{H}_{2}$ may be a potential therapy for sensorineural hearing impairment in AN patients.

\section{Abbreviations}

$\mathrm{ABR}$, auditory brainstem response; $\mathrm{AD}$, auditory dys-synchrony; AN, auditory neuropathy; CAP, compound action 
potential; DPOAE, distortion product otoacoustic emission; EDTA, ethylenediaminetetraacetic acid; $\mathrm{H}_{2}$, hydrogen gas; .OH, hydroxyl radicals; PBS, phosphate-buffered saline; ROS, reactive oxygen species; $\mathrm{RW}$, round window; SGN, spiral ganglion neuron; SPL, sound pressure level; TUNEL, terminal deoxynucleotidyl transferase-mediated dUTP-biotin nick-end labeling.

\section{Acknowledgements}

This work was supported by the State Key Program of the National Natural Science Foundation of China (No 30930098 to Jian-hua QIU), the Major State Basic Research Development Program of China (973 Program) (No 2011CB504505 to Jianhua QIU) and the Young Scientists Fund of the National Natural Science Foundation of China (No 30801287 to Juan QU; 81101409 to Ke-liang XIE).

\section{Author contribution}

Jian-hua QIU and Ke-liang XIE designed research; Juan QU, Yun-na GAN, Ke-liang XIE, Wen-bo LIU, Ya-fei WANG, and Ren-yi HEI performed research; Wen-juan MI and Jian-hua QIU contributed new reagents or analytic tools; Jian-hua QIU analyzed data; Juan QU, Ke-liang XIE, and Jian-hua QIU wrote the paper.

\section{References}

1 Rance G. Auditory neuropathy/dys-synchrony and its perceptual consequences. Trends Amplif 2005; 9: 1-43.

2 Starr A, Picton TW, Sininger Y, Hood $\sqcup$, Berlin Cl. Auditory neuropathy. Brain 1996; 119: 741-53.

3 Vlastarakos PV, Nikolopoulos TP, Tavoulari E, Papacharalambous G, Korres S. Auditory neuropathy: endocochlear lesion or temporal processing impairment? Implications for diagnosis and management. Int J Pediatr Otorhinolaryngol 2008; 72: 1135-50.

4 Ohsawa I, Ishikawa M, Takahashi K, Watanabe M, Nishimaki K, Yamagata $\mathrm{K}$, et al. Hydrogen acts as a therapeutic antioxidant by selectively reducing cytotoxic oxygen radicals. Nat Med 2007; 13: 688-94.

5 Cai J, Kang Z, Liu WW, Luo X, Qiang S, Zhang JH, et al. Hydrogen therapy reduces apoptosis in neonatal hypoxia-ischemia rat model. Neurosci Lett 2008; 441: 167-72.

6 Cardinal JS, Zhan J, Wang Y, Sugimoto R, Tsung A, McCurry KR, et al. Oral hydrogen water prevents chronic allograft nephropathy in rats. Kidney Int 2010; 77: 101-9.

7 Dole M, Wilson FR, Fife WP. Hyperbaric hydrogen therapy: a possible treatment for cancer. Science 1975; 190: 152-4.

8 Huang CS, Kawamura T, Toyoda Y, Nakao A. Recent advances in hydrogen research as a therapeutic medical gas. Free Radic Res 2010; 44: 971-82.

9 Huang Y, Xie K, Li J, Xu N, Gong G, Wang G, et al. Beneficial effects of hydrogen gas against spinal cord ischemia-reperfusion injury in rabbits. Brain Res 2011; 1378: 125-36.

10 Ji X, Liu W, Xie K, Qu Y, Chao X, Chen T, et al. Beneficial effects of hydrogen gas in a rat model of traumatic brain injury via reducing oxidative stress. Brain Res 2010; 1354: 196-205.

11 Kajiyama S, Hasegawa G, Asano M, Hosoda H, Fukui M, Nakamura $\mathrm{N}$, et al. Supplementation of hydrogen-rich water improves lipid and glucose metabolism in patients with type 2 diabetes or impaired glucose tolerance. Nutr Res 2008; 28: 137-43.

12 Xie K, Yu Y, Pei Y, Hou L, Chen S, Xiong L, et al. Protective effects of hydrogen gas on murine polymicrobial sepsis via reducing oxidative stress and HMGB1 release. Shock 2010; 34: 90-7.

13 Xie K, Yu Y, Zhang Z, Liu W, Pei Y, Xiong L, et al. Hydrogen gas improves survival rate and organ damage in zymosan-induced generalized inflammation model. Shock 2010; 34: 495-501.

14 Cai J, Kang Z, Liu K, Liu W, Li R, Zhang JH, et al. Neuroprotective effects of hydrogen saline in neonatal hypoxia-ischemia rat model. Brain Res 2009; 1256: 129-37.

15 Kikkawa YS, Nakagawa T, Horie RT, Ito J. Hydrogen protects auditory hair cells from free radicals. Neuroreport 2009; 20: 689-94.

16 Lin Y, Kashio A, Sakamoto T, Suzukawa K, Kakigi A, Yamasoba T. Hydrogen in drinking water attenuates noise-induced hearing loss in guinea pigs. Neurosci Lett 2011; 487: 12-6.

17 Schmiedt RA, Okamura HO, Lang H, Schulte BA. Ouabain application to the round window of the gerbil cochlea: a model of auditory neuropathy and apoptosis. J Assoc Res Otolaryngol 2002; 3: 223-33.

18 Lang H, Schulte BA, Schmiedt RA. Ouabain induces apoptotic cell death in type I spiral ganglion neurons, but not type II neurons. J Assoc Res Otolaryngol 2005; 6: 63-74.

19 Lang H, Schulte BA, Goddard JC, Hedrick M, Schulte JB, Wei L, et al. Transplantation of mouse embryonic stem cells into the cochlea of an auditory-neuropathy animal model: effects of timing after injury. J Assoc Res Otolaryngol 2008; 9: 225-40

20 Gorga MP, Johnson TA, Kaminski JR, Beauchaine KL, Garner CA, Neely ST. Using a combination of click- and tone burst-evoked auditory brain stem response measurements to estimate pure-tone thresholds. Ear Hear 2006; 27: 60-74..

$21 \mathrm{Li} \mathrm{J,} \mathrm{Wang} \mathrm{C,} \mathrm{Zhang} \mathrm{JH,} \mathrm{Cai} \mathrm{JM,} \mathrm{Cao} \mathrm{YP,} \mathrm{Sun} \mathrm{XJ.} \mathrm{Hydrogen-rich} \mathrm{saline}$ improves memory function in a rat model of amyloid-beta-induced Alzheimer's disease by reduction of oxidative stress. Brain Res 2010; 1328: 152-61.

22 Sun H, Chen L, Zhou W, Hu L, Li L, Tu Q, et al. The protective role of hydrogen-rich saline in experimental liver injury in mice. J Hepatol 2011; 54: 471-80.

23 Elmore S. Apoptosis: a review of programmed cell death. Toxicol Pathol 2007; 35: 495-516. 